\title{
Efeitos das isoflavonas sobre 0 miométrio de ratas adultas
}

\author{
Effects of isoflavones on the adult rat myometrium
}

Rejane Mosquette ${ }^{1}$, Milena Pires de Campos Luciano Gomes ${ }^{2}$, Ricardo Santos Simões ${ }^{3}$, Mauro Abi Haidar ${ }^{4}$, Manuel de Jesus Simões ${ }^{5}$, José Maria Soares Júnior ${ }^{6}$, Edmund Chada Baracat ${ }^{7}$

\section{RESUM0}

Objetivo: avaliar as alterações no miométrio de ratas após o tratamento com isoflavonas, com parando-as às dos estrogênios, por técnicas histológicas e morfométricas. Métodos: 28 ratas adultas castradas foram divididas, ao acaso, em quatro grupos que receberam: GPropi - propilenoglicol; GExtr10 - extrato de soja (10 mg/kg por dia); GExtr300 - extrato de soja (300 mg/kg por dia) e GEce - estrogênios conjugados eqüinos (Ece - $200 \mu \mathrm{g} / \mathrm{kg}$ por dia). Após 21 dias de tratamento, todos os animais foram sacrificados, os úteros retirados e pesados, e fragmentos dos cornos uterinos foram coletados e fixados em formol a $10 \%$ e processados para inclusão em parafina. Os cortes histológicos foram corados pela hematoxilina e eosina e examinados em microscópio acoplado a analisador de imagens para avaliação da espessura, número de vasos sanguíneos e de eosinófilos no miométrio. Os resultados obtidos foram submetidos à análise de variância (ANOVA), seguida pelo teste de comparações múltiplas de Tukey-K ram er. Resultados: as isoflavonas, na concentração de $300 \mathrm{mg} / \mathrm{kg}$, induziram aumento significante na espessura do miométrio (GExtr300=25,6 $\pm 5,0 \mathrm{~mm}$ ) em relação ao controle (GPropi=5,5 $\pm 0,5 \mathrm{~mm}$ ). O efeito desta alta é comparável ao efeito dos estrogênios (GEce=27,5 $\pm 7,9 \mathrm{~mm})$. Em doses baixas $(10 \mathrm{mg} / \mathrm{kg})$, os valores foram similares ao controle. Além disso, as isoflavonas (GExtr300) induziram aumento no número de vasos sangüíneos (GExtr300=10,2 $\pm 3,6 / \mathrm{mm}^{2} ; \mathrm{GPropi}=3,5 \pm 1,6$ vasos $/ \mathrm{mm}^{2}$ ) e de eosinófilos (GExtr300=4,3 $\pm 0,9 ;$ GPropi $=0,15 \pm 0,01$ eosinófilos $/ \mathrm{mm}^{2}$ ), igualmente com paráveis aos efeitos obtidos pelo tratamento com Ece $\left(\mathrm{GEce}=9,2 \pm 1,1\right.$ eosinophils $\left./ \mathrm{mm}^{2}\right)$. Conclusão: as isoflavonas em doses altas $(300 \mathrm{mg} / \mathrm{kg}$ por dia, durante 21 dias) têm efeito proliferativo no miométrio de ratas, semelhante ao dos estrogênios.

PALAVRAS-CHAVE: Isoflavonas; Estrogênios; Miométrio; Estudo experimental

\section{ABSTRACT}

Purpose: to evaluate histomorphom etric changes in the rat myometrium upon treatment with isoflavones, as com pared with estrogens, using histological and morphometric techniques. Methods: twenty-eight oophorectom ized adult rats were randomly divided into four treatment groups: GPropi = propylene glycol (control); GExtr10 - 10 mg/kg soybean extract; GExtr300 - 300 $\mathrm{mg} / \mathrm{kg}$ soy bean extract; GCee - $200 \mu \mathrm{g} / \mathrm{kg}$ conjugated equine estrogens (Cee). Drugs or drug vehicle were administered by gavage once a day for 21 days. Upon sacrifice, the uteri were removed and weighed. Fragments of uterine horns were collected and fixed in $10 \%$ formaldehyde and processed for paraffin inclusion. The histological sections were stained by hematoxylin and eosin and evaluated microscopically by means of an image analyzer to quantify the myometrial thickness and the number of blood vessels and eosinophils. The data were studied by analysis of variance (ANOVA) followed by the Tukey-Kramer multiple comparison test. Results: isoflavones in the concentration of $300 \mathrm{mg} / \mathrm{kg}$ induced a significant increase in the myometrium thickness $($ GExtr300 $=25.6 \pm 5.0 \mathrm{~mm}$ ) compared to control (GPropi $=5.5 \pm 0.5 \mathrm{~mm}$ ). The effect of this high dose is sim ilar to the estrogen effect $(\mathrm{GCee}=27.5 \pm 7.9 \mathrm{~m} \mathrm{~m})$. In low doses $(10 \mathrm{mg} / \mathrm{kg})$, the effect was similar to control. Isoflavones (GExtr300) induced also an increase in the number of blood vessels (GPropi $=3.5 \pm 1.6$; GExtr300 $=10.2 \pm 3.6$ vessels $/ \mathrm{mm}^{2}$ ) and of eosinophils $\left(\mathrm{CPropi}=0.15 \pm 0.01 ; \mathrm{GExtr} 300=4.3 \pm 0.9\right.$ eosinophils $\left./ \mathrm{mm}^{2}\right)$. These effects were comparable to those produced by Cee treatment in GCee (9.2 \pm 1.1 eosinophils $\left./ \mathrm{mm}^{2}\right)$. Conclusion: a high-dose treatment with isoflavones ( $300 \mathrm{mg} / \mathrm{kg}$ per day, $\left.21 \mathrm{days}\right)$ elicited an estrogen-like, highly significant proliferative action on the rat myometrium.

KEYWORDS: Isoflavones; Estrogens; Myometrium; Experimental study

\footnotetext{
Este projeto foi financiado pela FAPESP (Projeto $n^{\circ} 2003 / 13407-0$ ) (UNIFESP) São Paulo (SP), Brasil. Universidade de São Paulo (USP) São Paulo (SP), Brasil.

Correspondência: Rejane Mosquette

Disciplina de Histologia e Biologia Estrutural (UNIFESP/EPM)

Rua Botucatu, 740 - 04023-900 - São Paulo - SP - e-mail: rejane.morf@epm.br

1 Pós-Graduanda do Departamento de Morfologia da Universidade Federal de São Paulo (UNIFESP) São Paulo (SP), Brasil.

2 Bolsista de Iniciação Científica do CNPq do Departamento de Ginecologia da Universidade Federal de São Paulo (UNIFESP) São Paulo (SP), Brasil.

3 Médico Residente em Obstetrícia e Ginecologia da Universidade Federal de São Paulo (UNIFESP) São Paulo (SP), Brasil.

4 Prof. Livre-Docente e Chefe da Disciplina de Endocrinologia Ginecológica do Departamento de Ginecologia da Universidade Federal de São Paulo

5 Prof. Livre-Docente do Departamento de Morfologia da Universidade Federal de São Paulo (UNIFESP) São Paulo (SP), Brasil.

6 Prof. Adjunto da Disciplina de Endocrinologia Ginecológica do Departamento de Ginecologia da Universidade Federal de São Paulo (UNIFESP) São Paulo (SP), Brasil.

7 Prof. Titular do Departamento de Ginecologia da Universidade Federal de São Paulo (UNFESP/EPM) e Prof. Titular do Departamento de Ginecologia da 


\section{Introdução}

Nas últimas décadas, o número de mulheres que atingem a pós-menopausa tem se elevado consideravelmente. Este fenômeno está relacionado, entre outros, com a melhoria das condições de saúde pública e o aumento da expectativa de vida ${ }^{1}$. Nessa fase, há mudanças fisiológicas e clínicas decorrentes da falência ovariana, que se manifestam, precocemente, com os sintomas vasomotores (fogachos), sudorese, nervosismo, irritabilidade, insônia, cefaléia, vertigem, depressão, labilidade emocional, diminuição de capacidade de memorização, parestesias, formigamento, palpitações, mialgias, artralgias e diminuição da libido. Mais tardiamente, há atrofia da pele, distúrbios urogenitais e alterações ósteo-articulares ${ }^{2,3}$. Para minimizar essas alterações, tem-se empregado a terapia hormonal com esteróides sexuais, representados principalmente pelos estrogênios, de forma isolada ou em associação aos progestagênios. Esta terapia traz beneficios para a mulher, como diminuição dos sintomas, melhora da atrofia genital e prevenção de fraturas osteoporóticas ${ }^{4}$.

Apesar das vantagens da hormonioterapia, há aspectos negativos, como aumento do risco relativo de algumas neoplasias hormônio-dependentes, tais como de endométrio e de mama, bem como maior incremento do risco de doenças cardiovasculares e de tromboembolismo ${ }^{5}$. Além disso, as mulheres que fizeram uso da associação de estrogênio com progestagênio poderiam ainda ter outros efeitos colaterais indesejáveis, como mastalgia, aumento de peso corpóreo, alteração do perfil lipídico, etc. ${ }^{6}$.

Assim, devido a essas complicações, vários autores têm pesquisado fármacos que possam oferecer os efeitos benéficos dos estrogênios na pósmenopausa sem contudo apresentarem seus efeitos colaterais. Conseqüentemente, diversas substâncias têm sido utilizadas. Entre essas, incluemse os moduladores seletivos dos receptores de estrogênio (SERMs), como o tamoxifeno e o raloxifeno. Entretanto, esses fármacos apresentam algumas desvantagens, como reaparecimento ou aumento de fogachos e risco elevado de trombose. Assim, outras substâncias alternativas têm sido pesquisadas, como os fitoestrogênios, que poderiam agir sobre o receptor de estrogênio em virtude de sua similaridade estrutural química com os estrogênios ${ }^{7}$.

As isoflavonas podem agir como SERMs naturais ou fito-SERMs, visto que podem modular o receptor de estrogênio ${ }^{8}$. Esse fato ajudaria a compreender sua ação no alívio dos sintomas climatéricos, sobretudo nos fogachos, sem causar efeitos proliferativos no endométrio e na mama. Contudo, estudos recentes mostram que o uso prolongado de isoflavonas pode levar à proliferação endo- metrial em mulheres na pós-menopausa ${ }^{9}$.

A dieta rica em isoflavonas pode estimular o aumento do volume uterino. As isoflavonas (genisteína e daidzeína), na proporção de 0,3 a 0,55 $\mathrm{mg} / \mathrm{g}$ de alimento administrado a animais, causaram aumento do volume uterino em ratas castradas ${ }^{10}$. Verificou-se, também, que a ipriflavona, uma isoflavona sintética, não estimula o crescimento do leiomioma uterino em associação ao análogo do $\mathrm{GnRH}^{11}$. Contudo, não há trabalhos que demonstrem que as isoflavonas poderiam estimular o miométrio. Tal fato motivou-nos a realizar o presente estudo experimental. Desse modo temos por objetivo avaliar a ação de isoflavonas no útero de ratas tendo como comparação os estrogênios conjugados eqüinos.

\section{Métodos}

Utilizamos 40 ratas adultas (Rattus norvegicus albinus), virgens, de aproximadamente três meses, com peso de 180 a 210 gramas. Os animais foram fornecidos pelo Centro de Desenvolvimento de Modelos de Experimentação (CEDEME) da Universidade Federal de São Paulo (UNIFESP-EPM). Este projeto foi aprovado pelo Comitê de Ética da UNIFESP-EPM (Projeto $\mathrm{n}^{\circ}$. 091/03).

Todos os animais foram mantidos em biotério com condições de iluminação e temperatura ideais, alimentação com ração isenta de soja e água ad libitum. Os animais foram submetidos inicialmente a exame colpocitológico por 21 dias consecutivos e os que apresentaram ciclos estrais normais foram submetidos à ooforectomia bilateral. Após 28 dias do ato cirúrgico, 28 ratas foram divididas, ao acaso, em quatro grupos iguais, a saber: GPropi - receberam apenas o veículo (propilenoglicol); GExtr10 - receberam baixas doses de isoflavonas ${ }^{1}$ (10 mg/kg, por dia); GExtr300 - receberam altas doses de isoflavonas ${ }^{1}(300 \mathrm{mg} / \mathrm{kg}$, por dia), e GEce - receberam estrogênios conjugados eqüinos (Ece), na dose de $200 \mu \mathrm{g} / \mathrm{kg}$ por dia ${ }^{12}$.

As substâncias foram administradas por gavagem durante 21 dias consecutivos, sempre no mesmo período do dia (das 16:00 às 17:00 horas). As soluções foram diluídas em propilenoglicol, produzindo-se soluções homogêneas, de tal maneira que cada animal recebesse $1,0 \mathrm{~mL}$.

No último dia, logo após a administração das substâncias, todos os animais foram anestesiados com cetamina $(60 \mu \mathrm{g} / \mathrm{g})$ e xilazina $(16 \mu \mathrm{g} / \mathrm{g})$, por via intramuscular, e sacrificados, por decapitação, sendo o sangue colhido do tronco. Em seguida o soro foi separado e armazenado $\mathrm{a}-20^{\circ} \mathrm{C}$ para as determinações de estradiol e progesterona. Em seqüência foram retirados e pesados os úteros, sendo separados 
fragmentos do terço médio dos cornos uterinos de cada animal e imersos em formol a 10\% tamponado, para fixação, por um período de 12 horas.

Após a fixação, os fragmentos foram desidratados e processados para inclusão em parafina, segundo as técnicas habituais. A inclusão foi realizada de tal maneira que pudéssemos observar, nas lâminas histológicas, cortes transversais de cada corno uterino. Na seqüência, dos blocos foram obtidos cortes com $4 \mu \mathrm{m}$ de espessura, que foram corados pela hematoxilina e eosina.

A avaliação histológica e morfométrica foi realizada com o auxílio de um sistema de captura de imagens e análise pelo programa Axiovision 4 REL (Zeiss). Este processo consiste de um microscópio de luz (Axiolab Standard 20 da Carl Zeiss) acoplado a uma videocâmera de alta resolução (Zeiss), que transmite a imagem a um computador. Este procedimento foi realizado no Laboratório da Disciplina de Histologia da UNIFESP-EPM.

Para cada animal, foram feitas medições em 7 lâminas, tendo sido analisados os seguintes itens: espessura do miométrio, número de vasos sanguíneos e número de eosinófilos presentes no miométrio. A espessura do miométrio foi obtida medindo-se a distância da superfície interna da camada muscular circular até o limite externo da camada longitudinal, com o auxílio de uma linha reta, sendo feita uma medida em cada quadrante do corno uterino. Para a obtenção do número de vasos sanguíneos e de eosinófilos foram contados os números desses elementos observados em área de $1 \mathrm{~mm}^{2}$, na camada muscular circular.

Ao final do experimento foram dosados o estradiol e a progesterona no soro por radioimunoensaio, pelo método do duplo anticorpo (ICN Biomedicals Inc., Costa Mera, CA, USA). Estas dosagens foram realizadas no Laboratório Central do Hospital São Paulo, UNIFESP-EPM. As amostras foram dosadas em duplicata. A porcentagem máxima de reatividade cruzada destes kits é inferior a 0,01\%.

Para a análise dos resultados obtidos à microscopia de luz utilizou-se inicialmente a análise de variância (ANOVA). Quando houve significância, complementou-se com o teste de comparações múltiplas de Tukey-Kramer, com o propósito de observar se ocorreu diferença significante entre os grupos. Foi fixado em 5\% (p $\leq 0,05)$ o nível de significância para a rejeição da hipótese da nulidade.

\section{Resultados}

Não houve diferenças significantes entre os pesos corporais das ratas pertencentes aos vários grupos de estudo, ao final do experimento (peso médio de todos os grupos $220,1 \pm 18,9$ ). Com relação ao peso do útero observamos que as ratas tratadas com altas doses de isoflavonas ou de estrogênios apresentavam maior média de peso uterino (GExtr300=7,7 $\pm 2,9$ e GEce $=7,5 \pm 3,1 \mathrm{~g})(\mathrm{p}<0,01)$ em relação aos grupos GPropi e GExtr10 (GPropi=2,7 $\pm 0,5$ g e GExtr10=3,2 $\pm 0,8$ g). Não observamos diferenças entre os grupos nos níveis séricos de progesterona (média dos grupos 1,7 $\pm 0,7 \mathrm{ng} / \mathrm{mL}$ ). No GEce, como esperado, os níveis de estrogênios encontram-se bem elevados em comparação com os outros grupos (GEce $=32,1 \pm 6,3 \mathrm{pg} / \mathrm{mL}$, média dos outros grupos $=2,9 \pm 1,16 \mathrm{pg} / \mathrm{mL})(\mathrm{p}<0,001)($ Tabela 1$)$.

Tabela 1 - Pesos corporal e uterino e níveis séricos de estradiol e progesterona nos vários grupos estudados. Os resultados são média \pm desvio padrão de determinações em duplicata. Para cada grupo, $n=7$

\begin{tabular}{lcccc}
\hline & \multicolumn{4}{c}{ Grupos } \\
& GPropi & GExtr10 & GExtr300 & GEce \\
\hline Peso corporal $(\mathrm{g})$ & $238,5 \pm 24,4$ & $227,3 \pm 33,9$ & $202,0 \pm 13,8$ & $212,7 \pm 3,4$ \\
Peso uterino $(\mathrm{g})$ & $2,7 \pm 0,5$ & $3,2 \pm 0,8$ & $7,7 \pm 2,9 *$ & $7,5 \pm 3,1^{*}$ \\
Estradiol $(\mathrm{pg} / \mathrm{mL})$ & $2,5 \pm 0,9$ & $3,1 \pm 1,1$ & $3,4 \pm 1,5$ & $32,1 \pm 6,3^{*}$ \\
Progesterona $(\mathrm{ng} / \mathrm{mL})$ & $1,3 \pm 0,3$ & $1,7 \pm 0,9$ & $1,7 \pm 0,7$ & $2,1 \pm 1,1$
\end{tabular}

Os animais foram tratados durante 21 dias, por gavagem (volume final $=1 \mathrm{~mL}$ ), como segue: GPropi = propilenoglicol (controle); GExtr10 = isoflavonas $10 \mathrm{mg} / \mathrm{kg} ; \mathrm{GExtr300}$ = isoflavonas $300 \mathrm{mg} / \mathrm{kg}$; GEce = estrógenos conjugados eqüinos $200 \mathrm{mg} / \mathrm{kg} .{ }^{*} \mathrm{p}<0,01$ em relação ao grupo controle (GPropi)

As secções transversais dos cornos uterinos dos animais pertencentes ao GPropi (controle) apresentaram miométrio com espessura de $5,5 \pm 0,5 \mathrm{~mm}$, constituído por duas camadas de músculo liso bem definidas, uma circular (interna) e outra longitudinal (externa). As fibras musculares apresentaram núcleos pequenos e heterocromáticos, sendo que na região entre as camadas musculares observaramse inúmeros vasos sangüíneos (Figura 1A). O mesmo foi notado nos animais tratados com baixas doses de isoflavonas (GExtr10 $=5,4 \pm 1,4 \mathrm{~mm}$ ) onde a espessura não diferiu do grupo controle (GPropi $=5,5 \pm 0,5$ $\mathrm{mm}$ ). No entanto, os grupos tratados com altas doses de isoflavonas e estrogênios conjugados apresentaram miométrio bem mais espesso (GExtr300=25,6 $\pm 1,4$ $\mathrm{mm}$ e GEce $=27,5 \pm 7,9 \mathrm{~mm} ; \mathrm{p} \leq 0,001$ ), sendo que o miométrio possuía núcleos mais ricos em eucromatina e, entre as fibras musculares, numerosos eosinófilos (Figura 1 B, C e D).

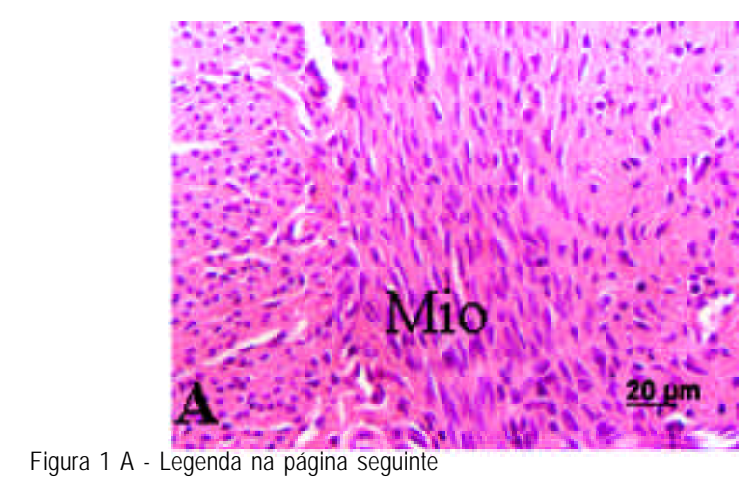

Rev Bras Ginecol Obstet. 2006; 28(4): 227-31 


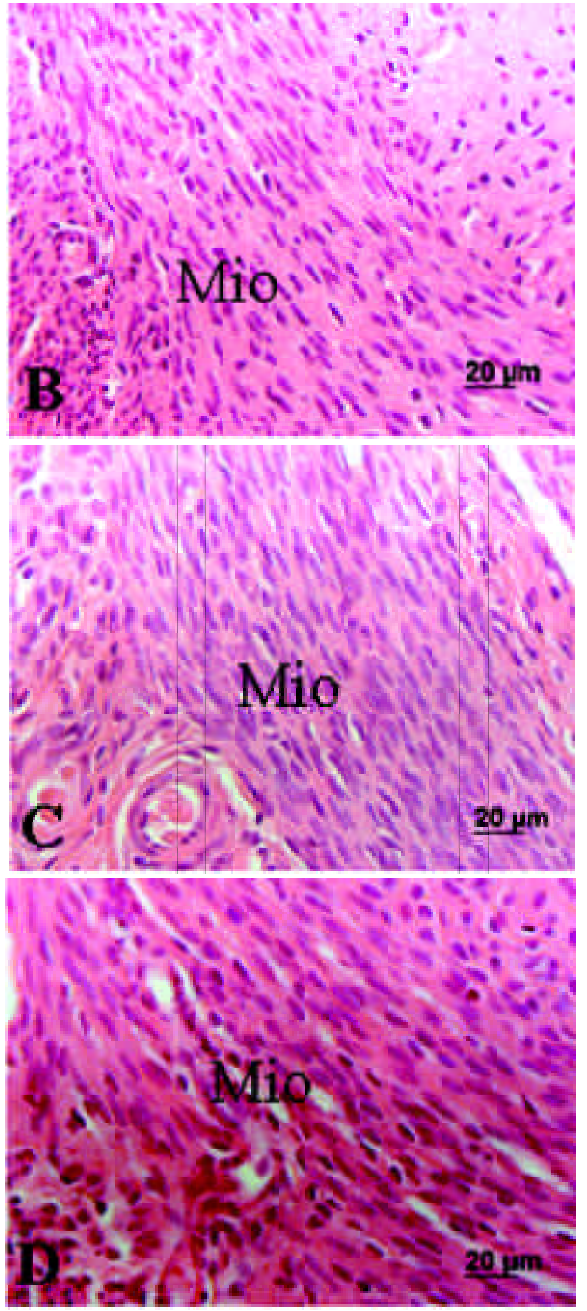

Figura 1 - Fotomicrografias mostrando cortes transversais dos úteros das ratas pertencentes aos vários grupos de estudo. Em A observar miométrio (Mio) de rata pertencente ao grupo controle; em B miométrio de rata tratada com isoflavona $10 \mathrm{mg} / \mathrm{kg} ; \mathrm{em} \mathrm{C}$ miométrio de rata tratada com isoflavona $300 \mathrm{mg} / \mathrm{kg}$ e em D miométrio de rata tratada com estrogênios $200 \mu \mathrm{g} / \mathrm{kg}(\mathrm{HE})$.

Notamos haver aumento significante do número de vasos sanguíneos no miométrio das ratas tratadas com altas doses de isoflavonas e estrogênios conjugados (GExtr300=10,2 $\pm 3,6 / \mathrm{mm}^{2}$ e GEce $12,9 \pm 3,9 / \mathrm{mm}^{2}$ ), sendo que o mesmo ocorreu em relação ao número de eosinófilos (GExtr300=4,3 $\pm 0,9$ / $\mathrm{mm}^{2}$ e GEce $=9,2 \pm 1,1 / \mathrm{mm}^{2}$ ) (Tabela 2 ).

Tabela 2 - Média e desvio padrão da espessura do miométrio, número de eosinófilos e de vasos sanguíneos presentes no miométrio nos vários grupos estudados.

\begin{tabular}{lcccc}
\hline & \multicolumn{4}{c}{ Grupos } \\
& GPropi & GExtr10 & GExtr300 & GEce \\
\hline $\begin{array}{l}\text { Espessura do } \\
\text { miométrio (mm) }\end{array}$ & $5,5 \pm 0,5$ & $5,4 \pm 1,4$ & $25,6 \pm 5,0^{*}$ & $27,5 \pm 7,9^{*}$ \\
$\begin{array}{l}\text { Número de vasos } \\
\text { sangüíneos/mm }\end{array}$ & $3,5 \pm 1,6$ & $4,9 \pm 1,7$ & $10,2 \pm 3,6 *$ & $12,9 \pm 3,9 *$ \\
$\begin{array}{l}\text { Número de eosinófilos/ } \\
\text { mm }^{2}\end{array}$ & $0,15 \pm 0,01$ & $0,28 \pm 0,02$ & $4,3 \pm 0,9 * *$ & $9,2 \pm 1,1^{*}$
\end{tabular}

GPropi = propilenoglicol (controle); GExtr10 = isoflavonas $10 \mathrm{mg} / \mathrm{kg} ; \mathrm{GExtr300}=$ isoflavonas $300 \mathrm{mg} / \mathrm{kg}$; GEce = estrógenos conjugados eqüinos $200 \mathrm{mg} / \mathrm{kg}$. ${ }^{*} p<0,001$ $\mathrm{e}{ }^{* \star} \mathrm{p}<0,01$ em relação ao grupo controle (GPropi).

\section{Discussão}

A ministração de esteróides sexuais, sobretudo estrogênios, na pós-menopausa constitui hoje motivo de grande preocupação, devido à estimulação endometrial e ao aumento do risco de câncer desta região. Além disso, há relatos do aumento do volume de leiomiomas uterinos após o tratamento hormonal ${ }^{13}$. Assim, o uso de substâncias com pouca ação estimulatória endometrial e miometrial seria importante para o clínico. Por este motivo, procuramos avaliar o efeito das isoflavonas no miométrio.

Verificou-se que a ministração de dieta rica em isoflavonas apresenta efeito trófico sobre o útero de $\operatorname{ratas}^{10}$. Contudo, em humanos, não há relatos quanto à ação dessas substâncias no leiomioma.

Diversos autores utilizaram em seus experimentos doses variadas e compostos com diferentes concentrações de genisteína e daidzeína. Além disso, as rações normalmente empregadas para criação e manutenção de animais em biotério apresentam resíduos de isoflavonas na sua composição ${ }^{10}$. Para nossa pesquisa foi confeccionada ração praticamente isenta de isoflavonas, pois não entrou na sua composição nenhum alimento que contivesse essas substâncias e que pudesse interferir em nossos resultados.

Acredita-se que a ação estimulatória das isoflavonas far-se-ia por sua ação no receptor de estrogênio, principalmente pela ativação do receptor beta ${ }^{14}$. Em apoio a esta hipótese, nossos dados mostraram haver grande número de eosinófilos, em especial nas áreas mais próximas ao miométrio, em ratas que receberam grande concentração do extrato de soja. Estudos prévios em nosso laboratório sugeriram que a presença de eosinófilos no útero de ratas depende da ativação do receptor de estrogênio ${ }^{15}$.

Sabe-se que os eosinófilos, no adulto, são formados na medula óssea e entram na circulação, onde permanecem por pequeno período de tempo ${ }^{16}$. A eosinofilia induzida pelo estrogênio é dificil de ser explicada, porém, os estrogênios ao que parecem teriam forte efeito de dilatação nos vasos uterinos, acelerando o crescimento do miométrio e do endométrio, o que faz supor que a sua presença no útero se correlaciona com o crescimento e remodelação deste órgão ${ }^{17}$. Assim, o efeito das isoflavonas em altas doses seria similar ao do estrogênio.

No extrato concentrado de soja, a genisteína e a daidzeína são referidas como as isoflavonas que apresentam maior ação estrogênica no útero de ratas. Estudos experimentais em ratas mostraram que doses entre 50 e $100 \mathrm{mg} / \mathrm{kg}$ ao dia de genisteína são efetivas para estimular o crescimento dos cornos uterinos e trofismo da citologia vaginal ${ }^{18}$. Outros autores reportaram ausência de atividade uterotrópica da genisteína quando sua concentra- 
ção fosse inferior a $2,5 \mathrm{mg} / \mathrm{kg}$ por dia, na alimentação de ratas castradas ${ }^{19}$. Em camundongas, doses maiores que $5 \mathrm{mg} / \mathrm{kg}$ por dia foram necessárias para se obterem efeitos uterotrópicos ${ }^{20}$.

Experimentalmente, notou-se que ratas Sprague-Dawley ${ }^{18}$ submetidas à administração de genisteína eram mais sensíveis do que as Wistar ${ }^{19}$. Responderam com doses de 50 e $100 \mathrm{mg} / \mathrm{kg}$ por dia de genisteína, apesar de não se detectar aumento da expressão do c-fos, um dos marcadores da atividade estrogênica. Contudo, no útero de ratas (DA/ Han) castradas foi relatada expressão aumentada desse marcador (c-fos) com a dose de $100 \mathrm{mg} / \mathrm{kg}$ por $\mathrm{dia}^{10}$. Nossos resultados mostraram que, com doses de $300 \mathrm{mg} / \mathrm{kg}$ por dia, houve alterações significativas no peso uterino e de modificações morfológicas e morfométricas no miométrio. Esta concentração corresponde a $25 \mathrm{mg} / \mathrm{kg}$ de genisteína, ou seja, um quarto da dose empregada no estudo anterior. É possível que outros compostos presentes no extrato poderiam atenuar o efeito da genisteína.

Nossos resultados mostraram que o extrato de soja em altas concentrações pode estimular o crescimento do miométrio de ratas adultas castradas, à semelhança do estrogênio, porém, em menor intensidade. Este fato, se extrapolado para a espécie humana, deve alertar o ginecologista para que tenha cuidado com a dose de isoflavona ministrada à paciente, como também com a duração da terapia.

\section{Referências}

1. Crawford SL. Epidemiology: methodologic challenges in the study of menopause. In: Lobo R, Kelsey J, Marcus $\mathrm{R}$, editors. Menopause: biology and pathobiology. San Diego: Academic Press; 2000. p. 159-74.

2. Han KK, Soares JM Jr, Haidar MA, de Lima GR, Baracat EC. Benefits of soy isoflavone therapeutic regimen on menopausal symptoms. Obstet Gynecol. 2002;99(3):389-94.

3. Baracat EC, Soares Júnior JM, Haidar MA, Rodrigues de Lima G. Aspectos reprodutivos no climatério. In: Fernandes $\mathrm{CE}$, coordenador. Consenso brasileiro multidisciplinar de assistência à saúde da mulher climatérica. São Paulo: SOBRAC; Rio de Janeiro: FEBRASGO; 2003. p. 251-3.

4. Pereira Filho AS, Soares Junior JM, Arkader J, Maciel GA, Baracat EC. Attitudes and practices about postmenopausal hormone therapy among female gynecologists in Brazil. Maturitas. 2005;51(2):146-53.

5. Manson JE, Hsia J, Johnson KC, Rossouw JE, Assaf AR, Lasser NL, et al. Estrogen plus progestin and the risk of coronary heart disease. N Engl J Med. 2003;349(6):523-34.

6. Chlebowski RT, Hendrix SL, Langer RD, Stefanick ML, Gass M, Lane D, et al. Influence of estrogen plus progestin on breast cancer and mammography in healthy postmenopausal women: the Women $\$$ s Health Initiative Randomized Trial. JAMA. 2003;289(24):3243-53.

7. Mosquette R, Simões MJ, Soares Júnior JM, Simões RS, Guerreiro da Silva IDC, Carvalho AM, et al. Mecanismos moleculares e efeitos das isoflavonas em mulheres. Arq Bras Fitomed Cient. 2004;2(1):21-9.

8. An J, Tzagarakis-Foster C, Scharschmidt TC, Lomri N, Leitman DC. Estrogen receptor beta-selective transcriptional activity and recruitment of coregulators by phytoestrogens. J Biol Chem. 2001;276(21):17808-14.

9. Unfer V, Casini ML, Costabile L, Mignosa M, Gerli S, Di Renzo GC. Endometrial effects of long-term treatment with phytoestrogens: a randomized, double-blind, placebocontrolled study. Fertil Steril. 2004;82(1):145-8.

10.Diel P, Smolnikar K, Schulz T, LaudenbachLeschowski U, Michna H, Vollmer G. Phytoestrogens and carcinogenesis-differential effects of genistein in experimental models of normal and malignant rat endometrium. Hum Reprod. 2001;16(5):997-1006.

11. Somekawa Y, Chiguchi M, Ishibashi T, Wakana K, Aso T. Efficacy of ipriflavone in preventing adverse effects of leuprolide. J Clin Endocrinol Metab. 2001;86(7):3202-6.

12. Baracat EC, Simões MJ, Soares Júnior JM, Haidar MA, Rodrigues de Lima G. determination of physiological doses of oral conjugated equine estrogens and medroxyporgesterone acetate in adult oophorectomized rats. Climacteric. 1999;2 Suppl 1:198.

13. Horn LC, Dietel M, Einenkel J. Hormone replacement therapy (HRT) and endometrial morphology under consideration of the different molecular pathways in endometrial carcinogenesis. Eur J Obstet Gynecol Reprod Biol. 2005;122(1):4-12.

14. Totta P, Acconcia F, Virgili F, Cassidy A, Weinberg PD, Rimbach G, et al. Daidzein-sulfate metabolites affect transcriptional and antiproliferative activities of estrogen receptor-beta in cultured human cancer cells. J Nutr. 2005;135(11):2687-93.

15.Simões MJ, Mora OA. Morphometric and ultrastructural aspect of macrophages present in the endometrium of rats during the estrous cycle. Rev Bras Biol. 1984;44(2):197-201.

16. Rytömaa T. Organ distribution and histochemical properties of eosinophil granulocytes in rat. Acta Pathol Microbiol Scand. 1960;50(Suppl 140):1-118.

17.Bjersing L, Borglin NE. Effect of hormones on incidence of uterine esosinophilia in rats. Acta Pathol Microbiol Scand. 1964;60(1): 27-35.

18.Santell RC, Chang YC, Nair MG, Helferich WG. Dietary genistein exerts oestrogenic effects upon the uterus, mammary gland and the hypothalamic/ pituitary axis in rats. J Nutr. 1997;127(2):263-9.

19. Makela S, Savolainen H, Aavik E, Myllarniemi M, Strauss L, Taskinen E, et al. Differentiation between vasculoprotective and uterotrophic effects of ligands with different binding affinities to estrogen receptors alpha and beta. Proc Natl Acad Sci USA. 1999;96(12):7077-82.

20.Ishimi Y, Arai N, Wang X, Wu J, Umegaki K, Miyaura $\mathrm{C}$, et al. Difference in effective dosage of genistein on bone and uterus in ovariectomized mice. Biochem Biophys Res Commun. 2000;274(3):697-701. 